\section{Speech therapy in palliative care and comfort feeding: current practice and way ahead}

\author{
Raymond Fong ${ }^{1}$, BSc, MPhil, Chun-Fung Tsai ${ }^{2}$, BSc, MMedSc, Wilfred Hing-Sang \\ Wong $^{3}$, PhD, Oi-Yan Yiu ${ }^{4}$, BSc, MMedSc, James Ka Hay Luk ${ }^{5}$, MBBS (HK), MSc \\ (Experimental Medicine) (UBC), FRCP (Edin), FRCP (Glasg), FRCP (Irel), FHKCP, \\ FHKAM (Medicine)
}

\begin{abstract}
Background. Speech therapy is important in palliative care and endof-life care of the geriatric population. Comfort feeding is an option for such patients, but there is no guideline for its operational and clinical practice. Thus, we surveyed the opinions of speech therapists on their role, clinical practice, and future development to formulate preliminary recommendations in dysphagia management and comfort feeding in patients on palliative care.

Methods. An online survey of speech therapists in Hong Kong was conducted between February and March 2018. The survey comprised 14 questions to assess speech therapists' understanding of palliative care, their current practice in comfort feeding, and their views on future development.
\end{abstract}

Results. Of 70 speech therapists invited, 38 (54\%) completed the survey. $89 \%$ of participants reported to have provided care to patients with dementia. The most common service provided was feeding and swallowing management (100\%), followed by education to patients, their family and/or the multidisciplinary team regarding communication and swallowing difficulties (88.2\%). There were diverse opinions on the practice of comfort feeding among speech therapists. Thus, $92.1 \%$ of participants agreed a need of development of practice guideline for palliative care.

Conclusions. The practice of comfort feeding varies among different speech therapists, particularly in the frequency of follow-up and the recommendation on liquid consistency. To improve the standard of care for patients receiving palliative care or end-of-life care, development of an evidence-based guideline for comfort feeding is recommended.

Key words: Deglutition disorders; Dementia; Feeding methods; Palliative care; Terminal care

\section{ORIGINAL ARTICLE}

\footnotetext{
Division of Speech Therapy, Department of Otorhinolaryngology, Head and Neck Surgery, Faculty of Medicine, The Chinese University of Hong Kong

2 Speech Therapy Department, Queen Elizabeth Hospital

3 Department of Paediatrics and Adolescent Medicine, Queen Mary Hospital, The University of Hong Kong

4 Speech Therapy Department, Hong Kong Children's Hospital

5 Department of Medicine and Geriatrics, Fung Yiu King Hospital
}

Correspondence to: $\mathrm{Mr}$ Raymond Fong, Room 303, Academic Building No. 2, The Chinese University of Hong Kong, Shatin, Hong Kong SAR.

Email: raymondfong@ent.cuhk.edu.hk

\section{INTRODUCTION}

Speech therapy can help rehabilitate patients with communication and swallowing problems secondary to neurological diseases or cancer. ${ }^{1}$ Patients with advanced and irreversible diseases receiving palliative/end-of-life care are in growing demand for speech therapy. Speech therapists should take on an active role in the multidisciplinary palliative care team. ${ }^{2}$ They can assist the medical team in advance care planning and end-of-life decisions regarding oral feeding or 
alternative nutrition and hydration options, mode of communication, and tracheostomy placement. ${ }^{3}$ Patients in palliative care may not opt for active medical interventions, and thus the goal of dysphagia management may be more of supportive than rehabilitative. ${ }^{4}$ In an international survey, 96\% (319/332) of respondents considered speech therapists to have a role in serving patients with life-limiting illness, but this area is under-resourced and poorly developed. ${ }^{5}$

Swallowing and feeding problems are prevalent among patients receiving palliative care. ${ }^{6}$ In the geriatric population, those with dementia often need palliative care, especially those with advanced disease. $84 \%$ to $93 \%$ of patients with moderate-tosevere dementia of Alzheimer's type are estimated to have dysphagia. ${ }^{7}$ In addition, $86 \%$ of nursing homes residents were reported to have swallowing and feeding problems. ${ }^{8}$ Tube feeding is often used but remains controversial, ${ }^{9}$ and thus comfort feeding is advocated. ${ }^{10,11}$ The European Society for clinical Nutrition and Metabolism guideline on ethical aspects of artificial nutrition and hydration defines comfort feeding as an individualised feeding care plan that can avoid negative connotations. ${ }^{10}$ It is elaborated as: "patients are fed as long as it is not distressing, and feedings are comfort oriented that are the least-invasive and potentially most satisfying way of attempting to maintain nutrition through careful hand feeding" ${ }^{12}$ However, the terminologies of feeding plan for patients receiving palliative care are inconsistent; an alternative of tube feeding has been mentioned as careful hand feeding, ${ }^{13}$ careful spoon feeding, ${ }^{14}$ oral-assisted feeding, ${ }^{15}$ and risk feeding. ${ }^{16}$ Non-standardised use of terminology may hinder knowledge exchange and create misunderstanding among multidisciplinary team members, ultimately affecting patient care.

Regarding the timing of evaluation and intervention intensity for dysphagia, stroke patients should receive a dysphagia assessment within 48 hours of stroke onset to ensure timely and appropriate recommendation about oral intake. ${ }^{17}$ The optimal intervention intensity in speech-language pathology has been studied. ${ }^{18}$ High-intensity swallowing therapy is more effective in patients with stroke. ${ }^{19}$ However, different speech therapists have differing practices in the intensity of therapy in patients with similar severity. ${ }^{20}$ This may be due to a lack of clinical trials or guidelines to provide recommendations on the optimal intervention intensity. Similarly, no such research has been conducted in the comfort feeding population.

There is no guideline on the responsibility of comfort feeding or on the roles of members of the multidisciplinary team. Regarding who is responsible for recommending comfort feeding, the guideline by Buckinghamshire Healthcare stated that "the consultant or general practitioner should make the ultimate decision about palliative feeding for comfort... multidisciplinary team discussions have taken place and adequate information has been shared with the patient/family". In addition, the responsibility of feeding the patient on a day-to-day basis is also ambiguous. It should be the hospital's staff responsibility if a family member is not present. Nonetheless, whether such responsibility is shared by different staff members of the hospital, and the legal liability that comes with feedingrelated complications need to be agreed among multidisciplinary team members. It is thus empirical to determine the current practice in order to make appropriate recommendations.

Instrumental assessment of silent aspiration and effectiveness of compensatory strategies during swallowing has been reported. ${ }^{21,22}$ However, study on instrumental assessment for those receiving comfort feeding and palliative care is lacking. The clinical decision currently rests on speech therapists, but there is no consensus on the effectiveness of swallowing strategies or instrumental assessment.

This study aimed to survey the opinions of speech therapists on their role, clinical practice, and future development to formulate preliminary recommendations in dysphagia management and comfort feeding in patients on palliative care.

\section{METHODS}

This was a cross-sectional survey of speech therapists in Hong Kong. It allowed examination of current situation by describing associated factors such as behaviours and attitudes, without influencing them in anyway. ${ }^{23,24}$ An online survey was designed based on the Tailored Design Method. ${ }^{25}$ Questions were formulated based on a literature review on palliative care and comfort feeding as well as clinical 
experience of local speech therapists. Questions were reviewed by two experienced speech therapists and amended based on their comments. There were 14 questions to assess speech therapists' understanding of palliative care, their current practice in comfort feeding in terms of operational and clinical decision, and their views on future development (TABLE $\mathbf{I}$ ). The survey was disseminated through email and instant-messaging.

Convenience sampling was used to recruit participants; consent was implied by completing the survey. No personal identifiable information was collected to ensure anonymity. Data were corrected between February and March 2018 and entered into a spreadsheet. Descriptive statistics were used for analysis. Responses to open questions were analysed qualitatively using thematic analysis.

\section{RESULTS}

Of 70 speech therapists invited, 38 (54\%) completed the survey (TABLE 2). On average, they reported a comfort feeding rate of $13.5 \%$ in the inpatient setting, $7.0 \%$ in the outpatient setting, and $5.6 \%$ in the community setting.

\section{Understanding of speech therapists' role in palliative care and comfort feeding}

$97.4 \%$ of the participants agreed that "The role of speech therapists in palliative care includes assessing and managing feeding and swallowing difficulties in order to improve patient comfort and eating satisfaction, and promote positive feeding interactions." $89.5 \%$ of participants agreed that "Patients will be fed as long as it is not distressing, and feedings are comfort oriented that are the least-invasive and potentially most-satisfying way of attempting to maintain nutrition through careful hand-feeding."

\section{Current practice in palliative care and comfort feeding: operation}

Participants reported that they provided palliative care most commonly to patients with dementia $(89.5 \%)$ and terminal malignancy $(89.5 \%)$, followed by those of old age with various medical problems $(78.9 \%)$, those with progressive neurological diseases $(71.1 \%)$, those with irreversible end-stage organ failures $(68.4 \%)$, and those with profound neurological damage (57.9\%).
Of 34 participants who reported provision of palliative care to patients with dementia, the most common service they provided was feeding and swallowing management (100\%), followed by education to patients, their family and/or the multidisciplinary team regarding communication and swallowing difficulties (88.2\%), advice on oral hygiene $(44.1 \%)$, communication management $(41.2 \%)$, and saliva management $(23.5 \%)$.

For feeding management under palliative care, the most common term the participants used or came across was comfort feeding (100\%), followed by careful hand feeding (84.2\%), oral pleasure (34.2\%), quality-of-life feeding (13.2\%), and pleasurable feeding $(2.6 \%)$.

All participants reported that they had come across scenarios in which patients and/or caregivers refused speech therapist recommendation of nonoral feeding, which was then labelled as comfort feeding.

$55.3 \%$ of participants reported that comfort feeding was recommended/ordered as a mutual consensus of speech therapists and medical team, whereas $34.2 \%$ reported that it was recommended by the clinical teams, and $10.5 \%$ reported that it was by speech therapists.

$97.4 \%$ of participants reported that family was responsible for comfort feeding of inpatients, whereas $42.1 \%$ reported that nurses and health care assistants were also responsible. However, $13.2 \%$ of participants reported no designated person in their workplace.

$47.4 \%$ of participants reported that they followed up patients with dysphagia on comfort feeding as frequent as other dysphagic patients, whereas $26.3 \%$ reported to follow up less frequently, $15.8 \%$ reported to follow up more frequently, and $10.5 \%$ reported no follow-up.

\section{Current practice in palliative care and comfort feeding: clinical decision}

$73.7 \%$ of participants reported that they would not perform instrumental assessment (eg, videofluoroscopic swallowing study) for patients on comfort feeding, because of (1) patient frailty, (2) not changing clinical decision, (3) radiation 
TABLE 1

Survey questions and responses

Question

$\%$ of participants

Section I: Understanding of speech therapists' role in palliative care and comfort feeding

1. Please state your level of agreement with the following statements (strongly agree, agree, neutral, disagree, and strongly disagree)

The role of speech therapists in palliative care includes assessing and managing feeding and swallowing difficulties in order to improve patient comfort and eating satisfaction, and promote positive feeding interactions. Patients will be fed as long as it is not distressing, and feedings are comfort oriented that they are the leastinvasive and potentially most-satisfying way of attempting to maintain nutrition through careful hand-feeding."

$97.4 \%$ agree or strongly agree $89.5 \%$ agree or strongly agree

Section II: Current practice in palliative care and comfort feeding: operation

2. Please indicate the patient groups that palliative care is provided in your workplace: (can select more than one)

Dementia

Progressive neurological diseases (eg, motor neuron disease, Parkinson's disease)

Profound neurological damage (eg, severe stroke, traumatic brain injury, brain or spinal cord injury)

Old age with various medical problems

Terminal malignancy

89.5

Irreversible end-stage organ failures (eg, chronic obstructive pulmonary disease, end-stage renal failure)

3. What aspects of care are you involved in when caring for individuals with dementia receiving palliative care? (can select more than one)

Feeding and swallowing management

Communication management

Advice on oral hygiene

Saliva management

Education of the patient, their family and/or the multidisciplinary team regarding communication and swallowing difficulties

Others, please specify:

4. In terms of feeding management under palliative care, what are the terminologies you use or come across: (can select more than one)

Comfort feeding

Careful hand feeding

Comfort hand feeding

Oral pleasure

Quality-of-life feeding

Others

5. Have you come across patients (and/or their caregivers) who refuse to follow speech therapist recommendation of non-oral feeding and then being labelled as 'comfort feeding'?

Yes

100

No

6. Who is in the position to recommend/order comfort feeding?

Medical team

Mutual consensus between speech therapists and medical team

Speech therapist

7. Who is/are responsible for feeding the patient under comfort feeding in an in-patient setting? (can select more than one)

Nurse

Patient care assistant

Family

No designated person

8. How is the follow-up of the majority of patients with dysphagia under comfort feeding compared to other dysphagic patients?

More frequently

Same 
TABLE 1 (cont'd)

\begin{tabular}{|c|c|}
\hline Question & $\%$ of participants \\
\hline \multicolumn{2}{|l|}{ Section III: Current practice in palliative care and comfort feeding: clinical decision } \\
\hline \multicolumn{2}{|l|}{ 9. Would you go for instrumental assessment eg, videofluoroscopic swallowing study? } \\
\hline Yes, I would tend to & 26.3 \\
\hline No, I would tend not to & 73.7 \\
\hline \multicolumn{2}{|l|}{ 10. Would you go for any form of active swallowing therapy eg neuromuscular electrical stimulation? } \\
\hline Yes, I would tend to & 7.9 \\
\hline No, I would tend not to & 92.1 \\
\hline \multicolumn{2}{|l|}{ 11. Would you suggest the use of any compensatory strategies / swallowing maneuvers? } \\
\hline Yes, I would tend to & 94.7 \\
\hline No, I would tend not to & 5.3 \\
\hline \multicolumn{2}{|l|}{$\begin{array}{l}\text { 12. For patients who demonstrated signs of aspiration across consistencies of food and liquid and is recommended } \\
\text { to have comfort feeding, what would be your most likely prescribed diet and fluid? }\end{array}$} \\
\hline \multicolumn{2}{|l|}{ Diet } \\
\hline No restriction on diet & 3.0 \\
\hline Soft/minced/congee diet & 55.0 \\
\hline Puree diet & 5.0 \\
\hline Fluid diet & 19.0 \\
\hline Would not prescribe diet & 18.0 \\
\hline \multicolumn{2}{|l|}{ Fluid } \\
\hline Thin fluid & 45.0 \\
\hline Thickened liquid using starch-based thickener & 2.0 \\
\hline Thickened liquid using xanthan-gum-based thickener & 3.0 \\
\hline Thickened liquid with no preference on thickener type & 34.0 \\
\hline Would not prescribe fluid consistency & 16.0 \\
\hline \multicolumn{2}{|l|}{ Section IV: Future development: comfort feeding } \\
\hline \multicolumn{2}{|l|}{ 13. What do you feel are the barriers/potential challenges to effective practice in this area? (can select more than one) } \\
\hline Resource availability (dedicated time and position / resource kit for family) & 92.1 \\
\hline Limited consensus among speech therapists & 71.1 \\
\hline Limited consensus among professionals in the workplace & 63.2 \\
\hline Limited coverage in training program of ST & 52.6 \\
\hline Further training constraints due to time/funding/lack of ongoing education course/ others, please specify: & 34.2 \\
\hline Legal consideration & 57.9 \\
\hline Ethical considerations & 39.5 \\
\hline \multicolumn{2}{|l|}{ 14. What do you feel would facilitate effective practice in this area? (can select more than one) } \\
\hline Development of practice guidelines for speech therapists in palliative care & 92.1 \\
\hline Education on speech therapists role in palliative care & 86.8 \\
\hline More collaboration with other professions & 76.3 \\
\hline Training on palliative care & 76.3 \\
\hline Additional research & 42.1 \\
\hline Colleagues' sharing of practice & 55.3 \\
\hline Others & 2.6 \\
\hline
\end{tabular}

exposure, and (4) limited resources. Nonetheless, $26.3 \%$ of participants reported that they would perform instrumental assessment (1) to document the physiology of swallowing for decision making by patients and caregivers, and (2) to recommend the safest and least-modified diet.

For active swallowing therapy (eg neuromuscular electrical stimulation), only three $(7.9 \%)$ participants reported that they would perform it for patients on comfort feeding, and $92.1 \%$ would not perform, because of (1) poor prognosis for this patient group, (2) general condition not suitable for therapy, (3) swallowing not a priority at this stage of life, and (4) limited resources.

$94.7 \%$ of participants would suggest compensatory strategy or swallowing manoeuvres to 
TABLE 2

Profile of participants $(n=38)$

\begin{tabular}{lc}
\hline Working experience & Value $^{*}$ \\
\hline Experience as speech therapists in medical setting, y & $9.91 \pm 8.06$ \\
Experience in working with patients under palliative care, y & $5.36 \pm 5.37$ \\
Service setting & \\
Acute inpatient service & $28(73.7)$ \\
Outpatient service & $29(76.3)$ \\
Rehabilitation inpatient service & $22(57.9)$ \\
Convalescent inpatient service & $13(34.2)$ \\
Community service & $7(18.4)$ \\
Service population & \\
Adults and children & $26(68.4)$ \\
Adults only & $11(28.9)$ \\
Children only & $1(2.6)$ \\
\hline * Data are presented as mean \pm standard deviation or no. (\%) of participants
\end{tabular}

patients on comfort feeding, because of (1) reduction of aspiration risk, (2) non-invasiveness, (3) easy to follow and execute, (4) enhancement of swallowing function, and (5) similar practice in all dysphagic patients. Two $(5.3 \%)$ participants would not suggest it, because patients were too weak to do so or could not follow.

If aspiration signs were present across all consistencies, $55 \%$ of participants would recommend soft/minced/congee diet, 19\% would recommend fluid diet, $18 \%$ would not prescribe diet, $5 \%$ would recommend puree diet, and 3\% would recommend no restriction on diet. In addition, $45 \%$ would recommend thin fluid, 34\% would recommend thickened liquid with no preference on thickener type, $16 \%$ would not prescribe fluid consistency, $3 \%$ would recommend thickened liquid using xanthangum based thickener, and 2\% would recommend thickened liquid using starch-based thickener.

\section{Future development}

Participants considered that the most common barrier to comfort feeding was a lack of resources (92.1\%) including time and position and resource kit for family, followed by limited consensus among speech therapists $(71.1 \%)$, limited consensus among professionals in the workplace $(63.2 \%)$, legal consideration $(57.9 \%)$, limited coverage in training programme of speech therapists $(52.6 \%)$, further training constraints $(34.2 \%)$, and ethical consideration (39.5\%).

To facilitate effective practice, participants most commonly opted for development of practice guideline for palliative care $(92.1 \%)$, followed by education on speech therapist role in comfort feeding $(86.8 \%)$, more collaboration with other professions $(76.3 \%)$, training on palliative care (76.3\%), and colleagues' sharing of practice (55.3\%). Other suggestions included additional research on this area $(42.1 \%)$ and overseas training (2.6\%).

\section{DISCUSSION}

\section{Role of speech therapists in palliative care}

$97.4 \%$ of speech therapists agreed that they should be involved in consultation with patients and families for management of communication and swallowing problems, as recommended by Pollens. ${ }^{2}$ In addition, all participants provided feeding and swallowing management, and $88.2 \%$ also provided education. Pollens recommended that speech therapists should optimise patient function related to dysphagia (eg, saliva management and oral hygiene) to improve patient comfort and satisfaction on feeding. ${ }^{2}$ However, only $44.1 \%$ of participants offered advice on oral hygiene and only $23.5 \%$ on saliva management, probably owing to the lack of role delineation. One critical role of palliative care 
is to maintain oral hygiene, ${ }^{26}$ but speech therapists are usually responsible for managing the risks of aspiration pneumonia. Poor oral hygiene is a risk factor for aspiration pneumonia. ${ }^{27}$ Thus, oral hygiene and saliva management should be an integral part of comfort feeding management.

\section{Terminologies}

The use of different terminologies for comfort feeding was common. Comfort feeding is defined as:"Patient will be fed as long as it is not distressing, and feedings are comfort oriented that they are the least-invasive and potentially most-satisfying way of attempting to maintain nutrition through careful hand-feeding." 12 The scope of comfort feeding is confined to patients with irreversible diseases receiving palliative care. The term 'careful hand feeding' should be used in the context of management strategy or technique instead of management approach. In Hong Kong, the Hospital Authority uses the term careful hand feeding officially with its definition described in the Guidelines on Life-sustaining Treatment in the Terminally Ill. ${ }^{28}$ The term should not be confused with 'risk feeding', which is defined as "providing oral intake despite high risk of aspiration", which can be adopted regardless of the clinical condition of the patient.

\section{Fluid and diet recommendations}

In patients with signs of aspiration present across all consistencies, $55 \%$ of participants would prescribe a soft/minced/congee diet, presumably to strike a balance between safety and quality of life, but only $3 \%$ would impose no restriction on diet. In addition, $45 \%$ would prescribe thin liquid and 39\% would prescribe thickened liquid. There is no guideline or clinical study on diet or liquid consistency most suitable for this patient population. As the principle of comfort feeding is continuation of oral feeding as long as the patient is comfortable and not distressed, diet and liquid recommendations should follow this principle. Nonetheless, speech therapists should be aware of the risk of aspiration and the patient reaction to persistent aspiration. An animal study showed that rabbits were more prone to pulmonary injury and fatality to chronic aspiration of thickened liquids using starch-based thickener than thin liquid and thickened liquid by gum-based thickener. ${ }^{29}$ This finding may provide preliminary evidence for speech therapists in prescribing liquid consistency to patients likely to aspirate. However, the Cochrane review showed that thickened liquids have positive immediate impact in preventing aspiration in patients with dementia, ${ }^{30}$ but the long-term effects were uncertain. Given the lack of evidence to support the use of thickened liquids in patients with dementia and potentially greater risk of pulmonary damage from thickeners, especially starch-based thickeners, prescribing thickened liquid in this patient population should be with caution.

\section{The way forward}

The only local guideline for comfort feeding is the Hospital Authority Guidelines on Life-sustaining Treatment in the Terminally Ill. ${ }^{28}$ However, speech therapists have differing practices in both operational and clinical aspects. The decision for comfort feeding should also be made by the multidisciplinary team instead of a single profession. In addition, 13.2\% of participants reported no designated person for comfort feeding patients in their workplace; such mismatch between clinical and operational aspects would affect the outcome. The clinical decisions of speech therapists on instrumental assessment, swallowing therapy, compensatory strategies, and swallowing manoeuvres were based on a collaborative practice by speech therapists rather than guidelines or clinical studies. Thus, an evidencebased guideline on comfort feeding is needed for patients, caregivers, speech therapists, and other members of the multidisciplinary team.

\section{CONCLUSION}

The practice of comfort feeding varies among different speech therapists, particularly in the frequency of follow-up and the recommendation on liquid consistency. To improve the standard of care for patients receiving palliative care or end-of-life care, development of an evidence-based guideline for comfort feeding is recommended.

\section{ACKNOWLEDGEMENT}

The authors would like to thank Lucie Ho and Danny $\mathrm{Au}$ as the reviewers of the initial questionnaire.

\section{DECLARATION}

The authors have no conflicts of interest to disclose. 


\section{REFERENCES}

1. American Speech-Language-Hearing Association. Scope of practice in speech-language pathology. Available at: www.asha. org/policy/. Accessed 29 August 2018.

2. Pollens RD. Integrating speech-language pathology services in palliative end-of-life care. Top Lang Disord 2012;32:13748. Crossref

3. Lambert H. The allied health care professional's role in assisting medical decision making at the end of life. Top Lang Disord 2012;32:119-36. Crossref

4. Chahda L, Mathisen BA, Carey LB. The role of speech-language pathologists in adult palliative care. Int J Speech Lang Pathol 2017;19:58-68. Crossref

5. O'Reilly AC, Walshe M. Perspectives on the role of the speech and language therapist in palliative care: an international survey. Palliat Med 2015;29:756-61. Crossref

6. Bogaardt H,Veerbeek L, Kelly K, van der Heide A, van Zuylen L, Speyer R. Swallowing problems at the end of the palliative phase: incidence and severity in 164 unsedated patients. Dysphagia 2015;30:145-51. Crossref

7. Affoo RH, Foley N, Rosenbek J, Shoemaker JK, Martin RE. Swallowing dysfunction and autonomic nervous system dysfunction in Alzheimer's disease: a scoping review of the evidence. J Am Geriatr Soc 2013;61:2203-13. Crossref

8. Mitchell SL, Teno JM, Kiely DK, Shaffer ML, Jones RN, Prigerson $\mathrm{HG}$, et al. The clinical course of advanced dementia. N Engl J Med 2009;361:1529-38. Crossref

9. Finucane TE, Christmas C, Travis K. Tube feeding in patients with advanced dementia: a review of the evidence. JAMA 1999;282:1365-70. Crossref

10. Druml C, Ballmer PE, Druml W, Oehmichen F, Shenkin A, Singer $\mathrm{P}$, et al. ESPEN guideline on ethical aspects of artificial nutrition and hydration. Clin Nutr 2016;35:545-56. Crossref

11. Hartigan I, Robinson S, O'Sullivan M, McLoughlin K, Gallagher $\mathrm{P}$, Timmons S. Palliative care for the person with dementia. Guidance document 4: Management of hydration and nutrition. Dublin: Irish Hospice Foundation; 2016.

12. Palecek EJ, Teno JM, Casarett DJ, Hanson LC, Rhodes RL, Mitchell SL. Comfort feeding only: a proposal to bring clarity to decision-making regarding difficulty with eating for persons with advanced dementia. J Am Geriatr Soc 2010;58:580-4. Crossref

13. Luk JK, Chan FH, Hui E, Tse CY. The feeding paradox in advanced dementia: a local perspective. Hong Kong Med J 2017;23:30610. Crossref

14. Campbell-Taylor I, Fisher RH. The clinical case against tube feeding in palliative care of the elderly. J Am Geriatr Soc 1987;35:1100-4. Crossref

15. Fischberg D, Bull J, Casarett D, Hanson LC, Klein SM, Rotella J, et al. Five things physicians and patients should question in hospice and palliative medicine. J Pain Symptom Manage 2013;45:595605. Crossref

16. The University of Auckland. Risk feeding guideline. New Zealand: University of Auckland. Available at: https://flexiblelearning. auckland.ac.nz/speech-science-dysphagia-education-hub/6/ files/riskfeedingguideline_post-endorsement.pdf. Accessed 29 August 2018.

17. Powers WJ, Rabinstein AA, Ackerson T, Adeoye OM, Bambakidis NC, Becker K, et al. 2018 Guidelines for the Early Management of Patients With Acute Ischemic Stroke: A Guideline for Healthcare Professionals from the American Heart Association/American Stroke Association. Stroke 2018;49:e46-e110. Crossref

18. Baker E. Optimal intervention intensity in speech-language pathology: discoveries, challenges, and unchartered territories. Int J Speech Lang Pathol 2012;45:478-85. Crossref

19. Carnaby G, Hankey GJ, Pizzi J. Behavioural intervention for dysphagia in acute stroke: a randomised controlled trial. Lancet Neurol 2006;5:31-7. Crossref

20. Krisciunas GP, Sokoloff W, Stepas K, Langmore SE. Survey of usual practice: dysphagia therapy in head and neck cancer patients. Dysphagia 2012;27:538-49. Crossref

21. Frowen JJ, Perry AR. Swallowing outcomes after radiotherapy for head and neck cancer: a systematic review. Head Neck 2006;28:932-44. Crossref

22. Kim SY, Kim TU, Hyun JK, Lee SJ. Differences in videofluoroscopic swallowing study (VFSS) findings according to the vascular territory involved in stroke. Dysphagia 2014;29:444-9. Crossref

23. Kelley K, Clark B, Brown V, Sitzia J. Good practice in the conduct and reporting of survey research. Int $\mathrm{J}$ Qual Health Care 2003;15:261-6. Crossref

24. Creswell JW. Research Design: Qualitative, Quantitative, and Mixed Methods Approaches. 4th ed. SAGE Publications; 2014.

25. Dillman DA. Mail and Internet Surveys: The Tailored Design Method, 2nd ed. New Jersey: Wiley; 2007.

26. Chalmers J, Pearson A. Oral hygiene care for residents with dementia: a literature review. J Adv Nurs 2005;52:410-9. Crossref

27. Luk JK, Chan DK. Preventing aspiration pneumonia in older people: do we have the 'know-how'? Hong Kong Med J 2014;20:421-7. Crossref

28. Hospital Authority. HA Guidelines on Life-Sustaining Treatment in the Terminally ill; 2015.

29. Nativ-Zeltzer N, Kuhn MA, Imai DM, Traslavina RP, Domer AS, Litts JK, et al. The effects of aspirated thickened water on survival and pulmonary injury in a rabbit model. Laryngoscope 2018;128:327-31. Crossref

30. Flynn E, Smith $\mathrm{CH}$, Walsh CD, Walshe M. Modifying the consistency of food and fluids for swallowing difficulties in dementia. Cochrane Database Sys Rev 2018;9:CD011077. Crossref 